\title{
Regulation of Chemokine Production by the Oxidative Metabolism of L-Arginine in a Human Mixed Lymphocyte Reaction'
}

\author{
Jonathan B. Orens, ${ }^{*}$ Nicholas W. Lukacs, $\uparrow$ Steven L. Kunkel,$\uparrow$ \\ Marie D. BurdicK, ${ }^{*}$ CAROL A. WilKE, ${ }^{*}$ Alfred WALZ, $\ddagger$ \\ AND ROBERT M. STRIETER ${ }^{*, 2}$

\begin{abstract}
Departments of ${ }^{*}$ Medicine, Division of Pulmonary and Critical Care and + Pathology, The University of Michigan, Ann Arbor, Michigan; and $\$$ The Theodor Kocher Institute, University of Bern, Bern, Switzerland
\end{abstract}

Received October 1, 1993; accepted February 6, 1994

\begin{abstract}
Previous studies have shown that during the development of a mixed lymphocyte reaction (MLR) levels of the chemotactic cytokines IL-8 and MCP-1 (members of the C-X-C and C-C supergene families, respectively) increase in a time-dependent fashion, and that the production of these chemokines correlates with the magnitude of responsiveness to alloantigen (13). Furthermore, the responsiveness to alloantigen in the context of a MLR has been shown to be regulated by the oxidative metabolism of L-arginine (12). We postulated that competitive antagonism of the L-arginine metabolic pathway in a human MLR may alter the production of members of the $\mathrm{C}-\mathrm{C}$ and $\mathrm{C}-\mathrm{X}-\mathrm{C}$ chemokine families. To test this hypothesis, mononuclear cells were isolated from healthy individuals and subjected to a one-way MLR in the presence or absence of varying concentrations of an L-arginine competitive inhibitor, $\mathrm{N}^{\mathrm{G}}$-methyl-L-arginine (NMA: 50 to 500 $\mu M)$. When the MLR was performed in the presence of NMA $(500 \mu M)$, the production of IL8 increased twofold $(P<0.05)$ and ENA-78 increased fivefold $(P<0.05)$, while MCP- 1 and MIP$1 \alpha$ were not significantly altered. These findings suggest that NMA, an inhibitor of the L-arginine metabolic pathway, may regulate the production of specific C-X-C chemokines, IL-8 and ENA78 , during a MLR. In contrast, the production of MCP-1 and MIP- $1 \alpha$, members of the $\mathrm{C}-\mathrm{C}$ chemokine family, does not appear to be regulated by this inhibitor of the oxidative metabolism of L-arginine in the context of a MLR. (-) 1994 Academic Press, Inc.
\end{abstract}

\section{INTRODUCTION}

Nitric oxide (NO) has been demonstrated to be important in the regulation of vascular tone, neurotransmission, and host defense mechanisms (1). Although the pathway for NO production has been well characterized, there have been difficulties in confirming the presence of inducible NO synthase in human cells (2-4). Furthermore, the exact mechanism for the role of $\mathrm{NO}$ in host defense remains controversial,

\footnotetext{
1 This research was supported in part by National Institutes of Health Grants HL50057, 1P50HL46487, HL02401, HL31693, and HL35276, and supported by a Research Training Fellowship Award from the American Lung Association.

${ }^{2}$ To whom correspondence and reprint requests should be addressed at Department of Internal Medicine, Division of Pulmonary and Critical Care Medicine, Box 0360, The University of Michigan Medical Center, 3916 Taubman Center, Ann Arbor, MI 48109-0360. Fax: (313) 764-4556. Dr. Strieter is a RJR Nabisco Research Scholar.
} 
particularly in humans. Despite these controversies, it is clear that the oxidative metabolism of L-arginine and subsequent production of NO is important in immunity and host defenses $(1,5)$.

The induction of a specific immune response is dependent upon a complex series of events which ultimately lead to the activation and proliferation of lymphocytes and the production of specific cytokines which are important in cellular activation and cellular recruitment. Although the mediators produced during an inflammatory response have been well characterized, the regulatory mechanisms responsible for the production of these mediators remain obscure. Of particular interest are the mechanisms which are responsible for the regulation of mediators which elicit specific leukocyte populations.

Two related families of chemotactic cytokines (chemokines) have been demonstrated to be important in the recruitment of various leukocyte populations, including neutrophils, monocytes, and lymphocytes (6-9). These chemokines include members of both the $\mathrm{C}-\mathrm{C}$ and $\mathrm{C}-\mathrm{X}-\mathrm{C}$ supergene families $(6,7)$. Members of the $\mathrm{C}-\mathrm{X}-\mathrm{C}$ family include interleukin 8 (IL-8), ${ }^{3}$ ENA-78, GRO- $\alpha$, GRO- $\beta$, GRO- $\gamma$, NAP- $2, \gamma$-IP 10 , and platelet factor 4 , while members of the $\mathrm{C}-\mathrm{C}$ family include monocyte chemoattractant protein (MCP-1), macrophage inflammatory proteins 1-alpha and 1-beta (MIP-1 $\alpha$, MIP-1 $\beta$ ), I-309, HC-14, and RANTES. As a group the C-X-C supergene family appears to be more important in eliciting neutrophils, while the $\mathrm{C}-\mathrm{C}$ family appears to be more important in monocyte and lymphocyte chemotaxis (6-9). Although investigators have observed the effect of various cytokines (IFN- $\gamma$, TNF $\alpha$ and $\beta$ ) on the production of NO $(10,11)$, there have been no studies documenting the affect of L-arginine metabolism on the production of chemokines.

Previous investigation has shown that in a mixed lymphocyte reaction (MLR) proliferation and CTL differentiation of rat splenocytes is regulated by the oxidative metabolism of L-arginine (12). In this study, the addition of $\mathrm{N}^{G}$ monomethyl-L-arginine (NMA), a potent competitive inhibitor of $\mathrm{L}$-arginine-dependent NO production, augmented proliferation and CTL responses in mixed lymphocyte cultures. Other studies have shown that at least two chemokines, IL- 8 and MCP-1, are produced in a timedependent fashion in the MLR (13).

We postulated that competitive antagonism of the $\mathrm{L}$-arginine metabolic pathway in a human MLR would alter the production of specific members of the $\mathrm{C}-\mathrm{C}$ and $\mathrm{C}-$ $\mathrm{X}-\mathrm{C}$ chemokine families. In this investigation, we demonstrate that NMA alters the normal expression of chemokines during the development of a human MLR.

\section{MATERIALS AND METHODS}

Cell isolation. Peripheral blood mononuclear cells (PBMC) were isolated as previously described (14). Briefly, heparinized venous blood was obtained from normal volunteers and diluted 1:1 with pyrogen-free normal saline. PBMC were isolated by density gradient centrifugation over Ficoll-Hypaque and washed three times. The mononuclear cells were a mixture of lymphocytes and monocytes, 72 and $28 \%$, respectively, and were $>95 \%$ viable by trypan blue exclusion. These cells were then

\footnotetext{
${ }^{3}$ Abbreviations used: NMA, $\mathrm{N}^{\mathrm{G}}$-methyl-L-arginine; ENA-78, epithelial neutrophil activating protein-78; IL-8, interleukin-8; MCP-1, monocyte chemoattractant protein-1; MIP- $1 \alpha$, macrophage inflammatory protein1 alpha.
} 
resuspended in culture media (RPMI, Hepes, $100 \mathrm{~m} M \mathrm{~L}$-glutamine, $100 \mathrm{U} / \mathrm{ml}$ penicillin, $100 \mathrm{ng} / \mathrm{ml}$ streptomycin, and $5 \%$ fetal calf serum).

Mixed lymphocyte response. PBMC were divided and designated as stimulator or responder cells. Stimulator cells were irradiated with 3000 Rads. For proliferation and chemokine analysis studies, $10^{5}$ stimulator and $10^{5}$ responder cells were mixed 1:1 in triplicate in 96-well microtiter plates (Costar, Cambridge, MA). NMA was added to the cultures in triplicate to obtain final well concentrations of $500,250,125$, and 50 $\mu M$, respectively. In additional experiments for chemokine analysis, stimulator $(5 \times$ $\left.10^{5}\right)$ and responder $\left(5 \times 10^{5}\right)$ cells were mixed 1:1 in 24-well tissue culture plates (Costar). All cultures were performed in triplicate and maintained at $37^{\circ} \mathrm{C}$ in humidified air $/ 5 \% \mathrm{CO}_{2}$. Supernatants were collected at Day 6 , the time at which maximal expression of chemokines in the MLR has been as shown by previous studies (13), and tested by specific ELISAs for IL-8, MCP-1, ENA-78, and MIP- $1 \alpha$. Proliferative activity was determined by adding $1 \mu \mathrm{Ci}$ of $\left[{ }^{3} \mathrm{H}\right]$ thymidine to each well at Day $516 \mathrm{hr}$ prior to the termination of the experiment. The cells were then lysed, labeled DNA was harvested using an automated cell harvester (PHD Cambridge, MA), and $\left[{ }^{3} \mathbf{H}\right]$ thymidine incorporation was quantitated. To compare multiple experiments using different donors, IL-8, ENA-78, MIP-1 $\alpha$, and MCP-1 levels were standardized within each experiment by dividing the level of antigen by the amount produced in the control (no exogenous NMA), thus giving an IL-8, ENA-78, MCP-1, and MIP- $1 \alpha$ index. Proliferation assays were standardized by dividing the $\left[{ }^{3} \mathrm{H}\right]$ thymidine uptake at each concentration of NMA by that of controls (no exogenous NMA).

Chemokine ELISAs. Antigenic IL-8, ENA-78, MCP-1, and MIP-1 $\alpha$ were quantitated from cell-free MLR supernatants using a modification of the double ligand method as previously described (13). Briefly, flat-bottomed 96-well microtiter plates (Nunc, Immuno-plate; Vanguard International, Neptune, $\mathrm{NJ}$ ) were coated with $50 \mu \mathrm{l} /$ well of rabbit anti-chemokine antibodies $\left[1 \mu \mathrm{g} / \mathrm{ml}\right.$ in $0.6 \mathrm{M} \mathrm{NaCl}, 0.26 \mathrm{M} \mathrm{H}_{3} \mathrm{BO}_{4}$, and 0.08 $N \mathrm{NaOH}\left(\mathrm{pH} \mathrm{9.6)]} \mathrm{for} 16 \mathrm{hr}\right.$ at $4^{\circ} \mathrm{C}$ and then washed with PBS (pH 7.5) and $0.05 \%$ Tween (wash buffer). This buffer was used to wash the plates throughout this assay. Microtiter plate nonspecific binding sites were blocked with $2 \%$ bovine serum albumin in PBS and incubated for $90 \mathrm{~min}$ at $37^{\circ} \mathrm{C}$. The plates were washed three times before MLR supernatants (NEAT and a dilution of $1: 10 ; 50 \mu \mathrm{l}$ ) were added and incubated for $1 \mathrm{hr}$ at $37^{\circ} \mathrm{C}$. The plates were washed three times, and $50 \mu \mathrm{l} /$ well of biotinylated rabbit anti-chemokine antibodies was added and incubated for $30 \mathrm{~min}$ at $37^{\circ} \mathrm{C}$. The plates then were washed three times before adding streptavidin-peroxidase conjugate (Bio-Rad, Richmond, CA). After washing three times, $100 \mu$ l of peroxidase substrate $[0.67 \mathrm{mg} / \mathrm{ml}$ orthophenylene dichloride (Dako, Carinteria, CA) in $25 \mathrm{~m} M$ citrate) phosphate, $\mathrm{pH} 5.0$, and $0.0002 \% \mathrm{v} / \mathrm{v}$ hydrogen peroxide] was added. The reaction was terminated, after incubation of the plates at room temperature to the desired extinction, with $50 \mu \mathrm{l} /$ well of $3 \mathrm{M} \mathrm{H}_{2} \mathrm{SO}_{4}$ solution. The plates were read at $490 \mathrm{~nm}$ in an ELISA reader. For standards, $50 \mu \mathrm{l} /$ well of recombinant IL-8, ENA-78, MCP-1, or MIP-1 $\alpha$ in $1 / 2 \log$ dilutions from $1 \mathrm{pg} / \mathrm{ml}$ to $100 \mathrm{ng} / \mathrm{ml}$ was used. These ELISAs demonstrated a linear detection of chemokines at concentrations greater than $10 \mathrm{pg} / \mathrm{ml}$.

Reconstitution experiments. To establish the specificity of NMA as a competitive inhibitor of L-arginine metabolism, further experiments were performed with the addition of exogenous $L$ - and D-arginine. Cell isolation and MLRs were prepared as above. L-Arginine hydrochloride (Sigma) was added to the MLR to obtain well concentrations of 5000,1000,100, and $10 \mu M$ (above the $1.2 \mathrm{~m} M$ L-arginine in the stock 
RPMI) each in the presence NMA (500-50 $\mu M)$ as described above. The same process was performed with D-arginine hydrochloride (Sigma). All reagents and media were assessed for endotoxin by a limulus ambebocyte lysate assay (Whittaker Bioproducts, Walkersville, MD) and found to be $<0.1 \mathrm{EU} / \mathrm{ml}$ of endotoxin contamination. Cytokine production and cell proliferation were measured and compared to controls.

Statistical analysis. Data were analyzed by a MacIntosh IIfx computer using Statview II statistical package (Abacus Concepts, Inc., Berkley, CA). Data are expressed as mean \pm SEM and compared using a two-tailed Student $t$ test. Data were considered statistically significant if $P$ values were less than 0.05 .

\section{RESULTS}

The effect of NMA on the production of MLR-derived chemokines. To determine if the production of cytokines in the MLR could be altered by the inhibition of Larginine oxidative metabolism, initial experiments (experimental $n=18$ ) were performed with and without varying concentrations of NMA $(500-50 \mu M)$. The concentration range of NMA was chosen based on previously published data $(12,15,16)$. Two representative members of the $\mathrm{C}-\mathrm{X}-\mathrm{C}$ chemokine family, ENA-78 and IL-8, as well as two members of the C-C chemokine family, MCP-1 and MIP- $1 \alpha$, were measured by specific ELISA from MLR supernatants at Day 6. The C-X-C chemokines ENA-78 and IL-8 were maximally expressed at Day 6 in the MLR with levels of 0.767 $\pm 0.210 \mathrm{ng} / \mathrm{ml}$ and $29.995 \pm 12.013 \mathrm{ng} / \mathrm{ml}$, respectively. The addition of NMA $(50-$ $500 \mu M$ ) to the MLR augmented ENA-78 and IL-8 in a dose-dependent fashion compared to controls. A dose of $500 \mu M$ NMA resulted in peak ENA-78 and IL-8 levels which were 5.5 and 2.5 times control, respectively. These results were statistically significant at $P<0.05$ for NMA $125-500 \mu M$. There was no statistical difference for ENA-78 and IL-8 compared to control in the presence of $50 \mu M$ NMA (Fig. 1). The C-C chemokines MCP-1 and MIP- $1 \alpha$ were maximally expressed at Day 6 in the MLR
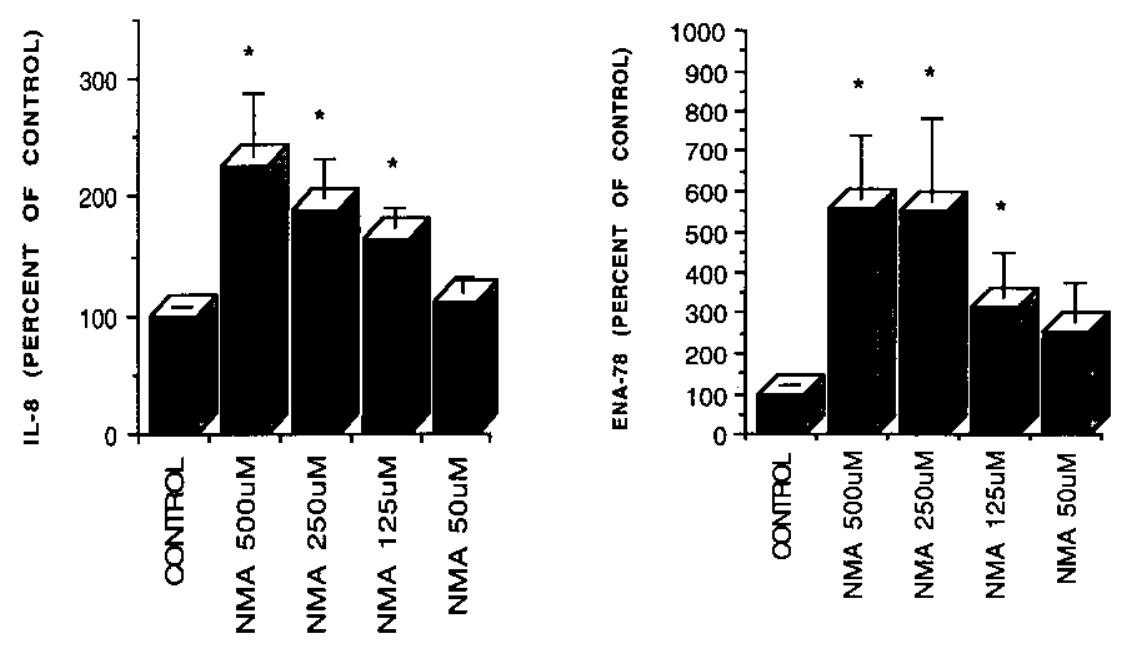

FIG. 1. The dose-dependent induction of the production of IL-8 and ENA-78 in response to NMA (50$500 \mu M$ ) as measured on Day 6 of the MLR. The values are expressed as a percentage of control (no NMA). ${ }^{*} P<0.05$. 
at levels of $2.673 \pm 0.956 \mathrm{ng} / \mathrm{ml}$ and $0.189 \pm 0.077 \mathrm{ng} / \mathrm{ml}$, respectively. These chemokines were not statistically altered in the MLR by the addition of NMA compared to controls (no NMA). These findings suggest that the inhibition of the L-arginine oxidative metabolism by the competitive inhibitor NMA was selective for augmenting the production of the $\mathrm{C}-\mathrm{X}-\mathrm{C}$ chemokines, but not $\mathrm{C}-\mathrm{C}$ chemokines.

Reconstitution with exogenous L-arginine. To determine whether the inhibition of L-arginine oxidative metabolism by the competitive inhibitor NMA could be attenuated with the addition of exogenous L-arginine, reconstitution studies were performed (experimental $n=18$ ). The addition of exogenous L-arginine in concentrations from 10 to $5000 \mu M$ to the MLR cultures in the presence or absence of NMA, failed to alter the stimulatory affect of NMA (500-50 $\mu M)$ on the production of ENA-78 and IL-8 measured at Day 6 (Fig. 2). Interestingly, the addition of exogenous L-arginine actually augmented the generation of both IL-8 and ENA-78 production in the MLR in the presence of NMA. The same experiments performed with D-arginine (experimental $n=4$ ) did not affect the changes caused by NMA alone (data not shown).

The effect of NMA on the proliferative response of the MLR. To test the role of NMA on the proliferative response of the MLR, NMA (500-50 $\mu M$ ) was added to the MLR as described above. Proliferation was assessed by incorporation of tritiated thymidine $(n=14)$. There was no significant increase in proliferation compared to control under all treatment conditions (Fig. 3).

\section{DISCUSSION}

The results of this study showed that NMA, a competitive inhibitor of L-argininedependent NO synthase, increases the levels of IL-8 and ENA-78 from human peripheral blood monocytes in the setting of an MLR. These two chemokines are members of the C-X-C supergene family, which are important in the recruitment of leu-
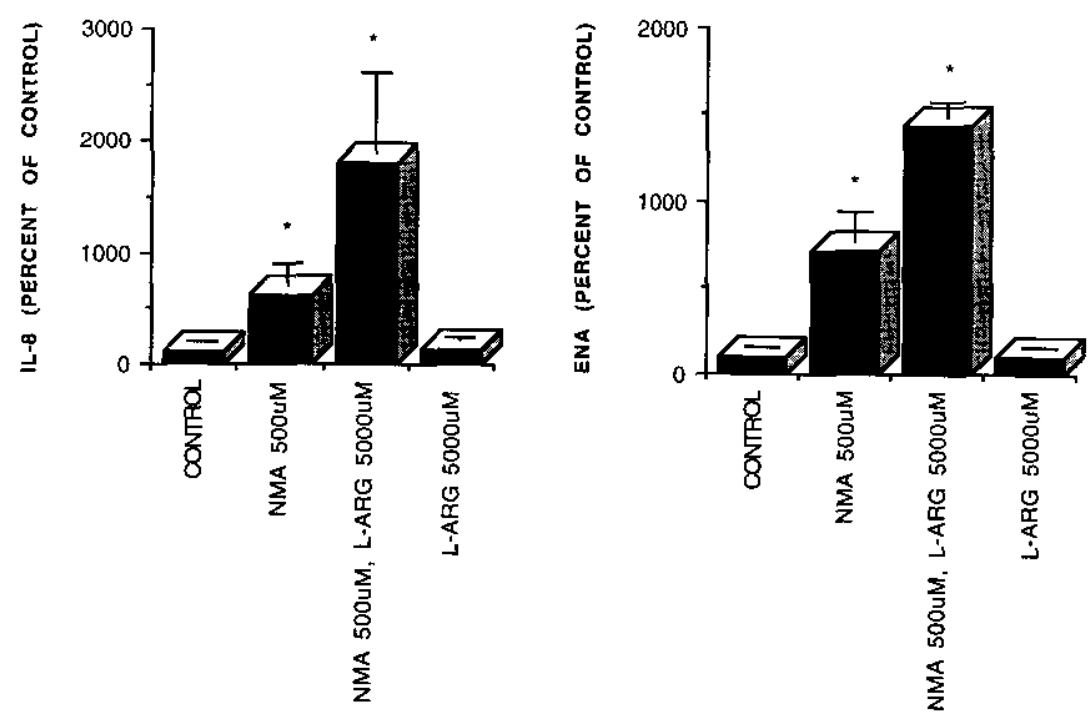

FIG. 2. The production of IL-8 and ENA-78 in response to control (untreated), NMA, NMA + L-ARG, or L-ARG. The values are expressed as a percentage of control on Day 6 of the MLR. ${ }^{*} P<0.05$. 


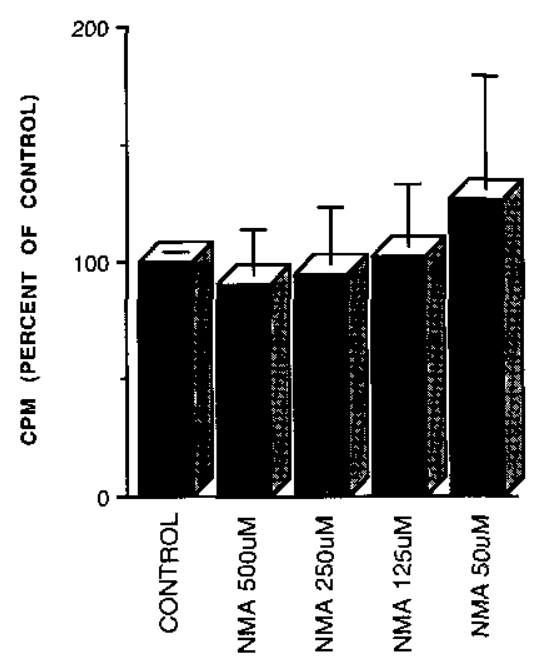

Fig. 3. Proliferative response of the MLR as measured by $\left[{ }^{3} \mathrm{H}\right] \mathrm{TdR}$ incorporation. NMA $(50-500 \mu M)$ failed to produce an increase in proliferation as compared to control (no treatment).

kocytes associated with acute inflammatory responses (polymorphonuclear leukocytes). In contrast, two representative members of the $\mathrm{C}-\mathrm{C}$ supergene family, MCP-1 and MIP- $1 \alpha$, were not altered by NMA in the MLR. These latter chemokines are most important in the recruitment of cells associated with chronic inflammatory responses (lymphocytes and mononuclear phagocytes). Augmentation of chemokine production with an inhibitor of L-arginine oxidative metabolism (NMA) suggests that L-arginine metabolism may play an inhibitory role on the production of $\mathrm{C}-\mathrm{X}-\mathrm{C}$ chemokines. This may be important in vivo since this may dictate the predominent cellular population recruited during the evolution of an inflammatory response.

There are several potential sites in the L-arginine pathway which may be responsible for the inhibition of IL- 8 and ENA-78. L-Arginine in the presence of NO synthase is converted into L-citrulline, nitrite, nitrate, and NO (17). As a result of this reaction there is upregulation of the second messanger, cyclic GMP. Any one or combination of these products may be responsible for the inhibitory effect on the production of IL8 and ENA-78. Interestingly, we were unable to reverse the effect of NMA by the addition of exogenous $\mathrm{L}$-arginine, at a concentration that was up to 10 times greater than that of NMA. Furthermore, L-arginine augmented the affect of NMA. L-Arginine without NMA had no effect on the production of these cytokines compared to control. These findings support the notion that NMA or the combination of NMA plus Larginine does not follow the "classical" pathway of inhibition of L-arginine metabolism in the augmented production of IL-8 and ENA-78.

Another interesting observation was the inability to demonstrate significant alterations in proliferation in our human MLR, despite the significant increase in production of IL-8 and ENA-78 from the same MLRs. In contrast, Hoffman and co-workers (12) documented significant increases in proliferation and the generation of CTL of rat splenocytes in MLRs cultured with NMA. Perhaps an explanation for this disparity is related to differences in species. There has been significant controversy in the literature regarding the existence of inducible NO synthase in human mononuclear phagocytes. Although some investigators believe that inducible NO synthase exists in humans, 
based on finding increased levels of nitrite and nitrate in the urine from patients with sepsis (18), clearcut proof is lacking. The difficulties in establishing inducible NO synthase in humans may be an artifact of in vitro models due to a lack of certain cofactors. Perhaps the disparite findings with reconstitution in our study are related to the current in vivo problem with inducible NO synthase. Other possibilities include alternative, yet undefined, pathways which incorporate L-arginine metabolism or direct effects of NMA on chemokine production.

This study has demonstrated that NMA, an L-arginine oxidative metabolism inhibitor, has stimulatory effects on the production of IL-8 and ENA-78, both members of the $\mathrm{C}-\mathrm{X}-\mathrm{C}$ chemokine family, from a human MLR. In contrast, NMA had no significant effect on the production of MCP-1 and MIP1- $\alpha$, members of the C-C chemokine family. Further investigation will be necessary to establish and better characterize the exact mechanism(s) involved in this regulation.

\section{REFERENCES}

1. Moncada, S., Palmer, R. M. J., and Higgs, E. A., Biochem. Pharmacol. 38(11), 1709, 1989.

2. Werner-Felmayer, G., Werner, E. R., Fuchs, D., Hausen, A., et al., Res. Immunol. 142, 555, 1991.

3. Mulsch, A., Res. Immunol. 142, 561, 1991.

4. Hibbs, J. B., Jr., Res. Immunol. 142, 565, 1991.

5. Liew, F. Y., and Cox, F. E. G., Immunol. Today 12(3), A17, 1991.

6. Schall, T. J., Cytokine 3(3), 165, 1991.

7. Walz, A., Burgener, R., and Carr, B., J. Exp. Med. 174, 1355, 1991.

8. Taub, D. D., Conlon, K., Lloyd, A. R., Oppenheirn, J. J., and Kelvin, D. J., Science 260, 355, 1993.

9. Wang, J. M., Sherry, B., Fivash, M. J., and Kelvin, D. J., J. Immunol. 150(7), 3022, 1993.

10. Stuehr, D. J., and Marletta, M. A., J. Immunol. 139, 518, 1987.

11. Stuehr, D. J., and Marletta, M. A., Cancer Res. 47, 5590, 1987b.

12. Hoffman, R. A., Langrehr, J. M., Billiar, T. R., Curran, R. D., and Simmons, R. L., J. Immunol. 145, $2220,1990$.

13. Christensen, P. J., Rolfe, M. W., Standiford, T. J., Burdick, M. D., Toews, G. B., and Strieter, R. M., J. Immunol. 151, 1205, 1993.

14. Strieter, R. M., Remick, D. G., Lynch, J. P., Genord, M., Raiford, C., Spengler, R., and Kunkel, S. L., Am. J. Respir. Cell Mol. Biol. 1, 57, 1989.

15. Denham, S., and Rowland, I. J., Clin. Exp. Immunol. 87, 157, 1992.

16. Mills, C. D., J. Immunol. 146, 2719, 1991.

17. Marletta, M. A., Yoon, P. S., Iyengar, F., Leaf, C. D., and Wishnok, J. S., Biochemistry 27, 8706, 1988.

18. Ochoa, J. B., Udekwu, A. O., Billiar, T. R., Curran, R. D., Cerra, F. B., Simmons, R. L., and Peitzman, A. B., Ann. Surg. 214, 621, 1991. 